\title{
DOCUMENTATION
}

\section{Rupture du barrage de l'Oued Fergoug (26 Novembre 1927)}

\author{
Rapport de la Commission technique chargée de déterminer les causes de la rupture
}

\section{Exposí:}

La Commission constituée par M. le Gouverneur Génèral en vue de déterminer les causes de la rupture lu barrage de l'Oued Fergoug, composée de :

M. Monser, Inspecteur Céneral des Ponts et Chaussées, Président de la $2^{e}$ Section du Conseil Ginéral des Ponts et Chaussées et de la Conmission des Grands Barrages, Président;

M. Vicarre, Inspecteur Général des Ponts et Chaussees, VicePrésident;

M. Deqove, Ingénicur en chef des Ponts et Chaussées, Secrétaire de la Commission des Grands Barrages; M. Chavanis, Ingénieur en chef des Ponts et Chaussées, adjoint all Directeur des Travaux publies des Chemins de fer et des Mines; M. VEn(iNiraud. Ingénieur en chef des Ponts et Chaussées; M. Scorto DI Vetrun, Ingénieur en chef des Ponts et Chaussées; 11. Sonotinar, Ingénicur en chef des Ponts et Chaussées; M. Balknst, Ingénieur en ehef des Ponts et Chaussées, Membres;

M. Bollaro. Ingénieur ordinaire des Ponts et Chaussces, attaché à la Direction des Travaux Publics, des Chemins de fer et des Nines, Rapporteur;

M. Drotnrs, Ingénieur ordinaire des Ponts et Claussées, Rapporteur Adioint,

a l'honneur de soumettre ses conchusions a M. le Gouverneur Général.

La Commission a siege du 19 au 27 décembre 1927 : elle a procédé à la visite des lieux le 20 décembre. Elle a étudié les documents existant dans les archives et relatifs à la construction du barrage ainsi qu'à sa réparation après la rupture de 1881 . Elle a reçu les dépositions des témoins oculaires de l'accidlent et pris comnaissance des observations sur le régime des pluies et des oueds alimentant le réservoir.

Elle croit devoir faire précćder d'un historique sommaire l'exposé des causes auxquelles elle attribue la rupture du barrage.

\section{Chapithe I. -- Histomete somaire.}

La mise en valeur de la plaine de l'Habra et de la Macta a été entreprise vers le milieu du siècle dernier par voie de concession de terres domaniales, avec obligation pour le concessionnaire de les assainir et de les irriguer au moven l'un barrageréservoir à établir à sés frais, risques et perils à l'aval immédiat du confluent de l'oued el Hammam, de loued Fergong et de loued Charzont. La construction du barrage dit de Yoied Fergoug, ou de l'llabra, on encore de Perregaux, fut fate par la Sociéte béneficiaire de la concession, sur un avant-projet dresse par l'Arhministration et annexé au cahier des charges de la concession; le projet définitif fut présenté par le concessionnaire en 1867 , en application des clauses du cahier des charges.

Le barrage primitif comportait un déversoir de 125 mètres de long, un mur ale $316 \mathrm{~m}$. 6 dit "grand mur " ct un mur dit "petit mur a de 30 mètres de long faisant aver le précétent un angle de $120^{\circ}$ environ et formant retour vers lamont; lancrage dans la rive droite n'avait pas part devoir etre réalise dans le prolongement du grand mur. Le déversoir thait arase a la cote 32 audessus de litiage conventiomel, situé lui-mème à 2 netres au-dessus du fond de loned. le couromement du barrage. a la cote 33.60 le courommement du parapet, a la cote 36 .

Les travaux étaient à peine achevés que le 10 mars 1872 . Te déversoir rut emporté sur une cinquantaine de nètres par une crue dont le débit a été évalué alors à $700 \mathrm{~m}^{3}$, la première rois semble-t-il. que le barrage était completement mis on clarge. Cet accident fut attribuć à l'insiffisance des fondations du déversoir.
Le déversoir fut reconstruit dans de meilleures conditions; après quoi, les travaux furent reçus définitivement le 7 avril 1873. Le procès-verbal de réception fut approuvé par le Gouverneur Général après avis du Conseil du Gouvernement, le 3 février 1874. Depuis sa réfection, le déversoir s'est bien comporté et n'a subi aucune avarie. Il s'agissait done d'un accident localisé, auquel if a été remédié d'une facon définitive.

La partie du barrage voisine de l'angle du grand et du petit mur ne tarda pas à inspirer des inquiétudes en raison de fissures, peu profondes d'ailleurs, qui se manifestèrent sur le couronnement et d'une tenilance au déversement du parement aval du grand mur. Ce déversement atteignait $0 \mathrm{~m} .21$ lorsque le 15 décembre 1881, à 5 heures du matin, une crue ouvrit dans le mur du barrage, une brèche d'environ 125 mètres de long, intéressant une partie du petit mur et une partie du grand mur; cette brèche était de forme trapéző̈dale en élévation; transversalement à l'ouvrage. la ligne de rupture affectait à l'aval, l'allure générale des courbes de cisaillement. La berge, rive droite immédiatement à l'aval, fut aussi emportée.

La hauteur d'eau au-dessus du déversoir au moment de la rupture, fut évaluée à $2 \mathrm{~m}$. 25, correspondant à un débit de $800 \mathrm{~m}^{3}$ sur le déversoir auxquels il faut ajouter $50 \mathrm{~m}^{3}$ passant par le seul évacuateur ouvert.

L'étude des causes de la rupture fut confiée d'une part, à un Ingénieur en chef des Ponts et. Chaussées et à un Ingénieur en chef des Mines, désignés comme experts par le Conseil de Préfecture d'Oran devant lequel l'Etat avait introduit une instance pour faire consacrer la responsabilité de la Société, d'autre part à une Commission d'Ingénieurs des Ponts et Chaussées, désignés par le Ministre de l'Agriculture ; cette Commission était présidée par M. l'Inoénieur en Chef Stoecklin.

Il fut reconnu que la cause fondamentale de la rupture a été I'insuffisance des fondations de la rive droite; la structure du terrain comportait des bancs alternés de grès et d'argile ayant un fort pendage vers l'aval et traversés de failles, ce qui a permis d'expliquer des glissements dans le sol d'appui; on n'avait d'ailleurs pas pris la précaution d'ancrer au moyen de redans les maçonneries assises sur les bancs de grès.

la reconstruction du barrage fut aussitot mise à l'étude et devant le refus de la Société, l'Administration des Ponts et Chaussées en fut chargée, la répartition des dépenses étant réservée; cette question a été ultérieurement tranchéc par le Conseil d'Etat (arrèt du 6 décembre 1895 ).

- Comme la partic du mur barrage restée intacte comportait un coefficient de sécurité du même ordre que celui des grands barrages analogues alors en service, elle fut conservée; l'on se borna à ferıner la brèche, à doubler le mur en retour par un prolongement du grand mur dans la rive droite et à protéger cette rive vers l'amont par un perré. Les ingénieurs chargés de la réparation, avaient dabord proposé de maintenir sans changement le profil de l'ouvrage dans la partie à reconstruire; à la suite des observations de la Commission de l'hydraulique agricole, le profil fut légèrement renforcé sur une certaine longueur dans la partie reconstruite.

Le barrage remis en service en 1885 , fut soumis accidentellement en 1000 , à une épreuve exceptionnelle. Des branchages et broussailles arrètés sur le déversoir par les fermettes de la passerelle, espacées de 3 mètres seulement, relevèrent le plan d'eau à $3 \mathrm{~m} .22$ au-dessus du déversoir, sans que le barrage, qui subit seulement de petites dégradations locales, présentât des traces de fatigue.

Pour prévenir le retour de cet incident, l'on espaça les fermettes de 6 mètres; l'engorgement du déversoir ne s'ent pas reproduit.

D'apres les evaluations tres approximatives qui ont pu ètre faites, le débit maximum de loued el Hammam et des autres. 
oneds alimentant le réservoir, était comparable au débit constaté lors de la rupture de 1881. La hauteur d'eau sur le déversoir, s'il ne s'était pas obstrué, aurait été elle-mème de l'ordre de 3 mètres, comme en 1881.

Depuis lors, le barrage n'eut pas à subir de crues élerant le plan d'eau sur le déversoir à une cote supérieure à $1 \mathrm{~m}$. 20, cote atteinte en 1904.

Il ne s'est rien passé d'anormal jusqu'au 26 novembre 1927 , date à laquelle le barrage a été rompu à $11 \mathrm{~h}$. 20 du matin ; lépaisseur de la lame d'eau sur la crête du déversoir a atteint $3 \mathrm{~m} .85$, au moment de la rupture.

\section{Chapitre II. - Crue des 25-26 novembre 1927.}

Le caractère anormal et tout à fait imprévu de cette crue, qui a entraîné la rupture du barrage, est bien marqué par l'importance du désastre qui a atteint toute une région de l'Algéric s'étendant sur une grande partie du département d'Oran et du département d'Alger. Sur la mème rivière de l'oued el Hammam et en amont du barrage, tous les ponts sont emportés ou gravement endommagés : le pont de Dublineau, sur la route nationale $N^{\circ} 6$ est complètement détruit; le pont des Trois rivières, sur la route nationale $N^{\circ} 7$, entre Mascara et Sidi-belAbbès, a ses rampes d'accès enlevées; le pont du chemin de G. C. $\mathrm{N}^{\circ} 1$ entre Mercier-Lacombe et Bou-Hanifia est emporté, le pont de la Ghethna, sur le chemin de fer d'Arzew à Colomb-Béchar, entre Dublineau et Tizi, est empoité; les viaducs d'accès des deux autres ouvrages sur la mème ligne subissent le mème sort.

En dehors du bassin de l'oued el Hammam, des dégâts très graves se produisent. Sur la route 11 d'Alger à Mostaganem par le bord de la mer, sur plus de 200 kilomètres, la route est coupće par des ensablements très importants, presque tous les ponts sont endommagés, 5 grands ponts s'effondrent. Sur la route 19 d'Orléansville à Ténès, un pont de 12 mètres est emporté. La route $t$ entre Mostaganem et Oran est coupée par des apports de terre considérables entre Mostaganem et la Stidia, et complètement submergée au pont de la Macta. Dans la nuit du 25 au 26 la ligne de chemin de fer d'Alger à Oran a été submergée à son passage à l'extrémité de la plaine de l'Habra, près de BouHenni. Le train de nuit d'Oran à Alger est resté bloqué par l'inondation et les voyageurs n'ont pu être repris que le 27 novembre par un train de secours venant d'Oran.

Sur la ligne d'Orléansville à Ténès, la voie a été bouleversée sur près de 11 kilomètres, 4 ponts de 20 à 25 mètres ont été emportés.

A Mostaganem, la petite rivière de l'Ä̈n-Sefra qui traverse la ville dans un aqueduc couvert, a èté gonflée d'une manière telle que l'aqueduc s'est engorgé. L'eau s'est accumulée à la tète de l'aqueduc et a entraîné dans le port, dans la nuit du 26 au 27 novembre, tout un quartier de la ville. Près de 150 personnes ont péri dans cette catastrophe. Le port de Mostaganem a été partiellement ensablé.

Pour le bassin de l'oued el Hammam, on trouvera ci-dessous en millimètres les hauteurs d'eau relevées aux différents pluviomètres du 24 au 26 novembre, avec cette remarque que les mesures sont faites à 6 heures du soir et qu'ainsi la hauteur d'eau indiquée pour la journée du 26 est en réalité la hauteur d'eau tombée entre le 25 à 6 heures du soir et le 26 à la mème heure. Ces chiffres ont été donnés par le Service Météorologique rattaché à l'Université d'Alger.

\begin{tabular}{|c|c|c|c|c|c|}
\hline Désignation & 24 & 25 & 26 & Total. & $\begin{array}{c}\text { TraNChe } \\
\text { ANNUELLE } \\
\text { MOYENNE } \\
\text { DE } 10 \text { ANS, } \\
1-9,1914, \\
31-8-1924\end{array}$ \\
\hline Oued Fergong barrage.. & 14 & 16 & 160 & 190 & 439 \\
\hline Mascara............. & 27 & 42 & 157 & 226 & 491 \\
\hline Aïn-Fékan .. & 23 & 23,7 & 149,2 & 195,9 & 385 \\
\hline Thiersville.......... & 17,2 & 20,1 & 69,5 & 106,8 & 446 \\
\hline Sidi-Ali (pr. Thiersville). & 15,8 & 23,8 & 77,0 & 116,6 & $n$ \\
\hline Mercier-Lacombe....... & 7,2 & 19,6 & 61,8 & 88,6 & 437 \\
\hline Oued Taria ...... & 23,0 & 17,0 & $n$ & 117 & 430 \\
\hline Franchetti & 31,1 & 32,8 & 92 & 115,9 & 486 \\
\hline Saïda.......... & 16,4 & 35,4 & $f i 1,2$ & 116 & 433 \\
\hline Aïn-el-Hadjar. . & 17,1 & 42,5 & 73,5 & 133,1 & " \\
\hline Tirman ........ & 9 & 26 & 25 & 51 & 333 \\
\hline
\end{tabular}

Une observation supplémentaire étant faile à 7 heures du matin, on a pu avoir d'autre part les hauteurs d'eau tombées dans la nuit du 25 au 26 (période de treize heures). Les chiffres les plus intéressants sont les suivants :

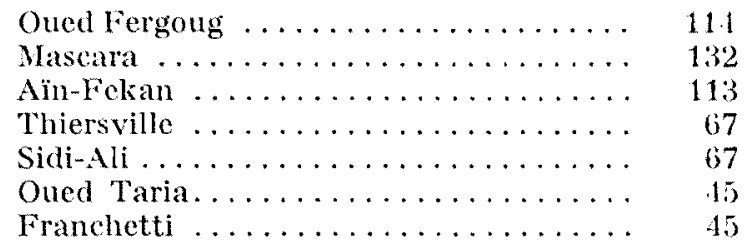

On constate qu'a Mascara par exemple la hauteur d'eau tombée en 3 jours est près de la moitié de la tranche annuelle moyenne et que la hauteur d'eau tombée dans la seule nuit du 25-26, représente pour plusieurs stations près du $1 / 3$ de la moyenne ammuelle. On constate aussi que les pluies exceptionnelies se sont étenclues sur la presque totalité de ce tres grand bassin qui comprend environ en amont du barrage, dix mille kilometres earrés. On s'est done bien trouvé en présence de précipitations d'une importance absolument imprévue et d'une étendue également sans précédent depuis l'occupation de l'Algérie.

En ce qui concerne le barrage, les observations faites a partir du 25 novembre 1927 , à 13 heures, heure à laquelle la crue commençant réllement, l'ordre fut donné au garde buragiste d'ouvrir en grand les vammes des évacuateurs, sont reproduites ciaprès :

\begin{tabular}{|c|c|c|c|}
\hline Jotrixites & HEURE & $\begin{array}{c}\text { Corte bu pax } \\
\text { D'bay }\end{array}$ & OBSERMTHOSS \\
\hline 25 novembre 1927 & $\begin{array}{l}13 \mathrm{h.} . \\
16 \mathrm{h.} \\
20 \mathrm{h.} 30\end{array}$ & $\begin{array}{l}8 \mathrm{~m} . \\
12 \mathrm{~m} . \\
20 \mathrm{m.} 30\end{array}$ & \\
\hline 26 novembre 1927 & $\begin{array}{l}0 \mathrm{~h} . \\
6 \mathrm{~h} . \\
7 \mathrm{~h} . \\
9 \mathrm{h.} . \\
10 \mathrm{h.} \\
11 \mathrm{h.} 20\end{array}$ & $\begin{array}{l}32 \mathrm{m.} .(1) \\
32 \mathrm{~m} .60 \\
34 \mathrm{~m} .10 \\
34 \mathrm{~m} .80 \\
35 \mathrm{~m} . \\
35 \mathrm{~m} .85 \text { (2) }\end{array}$ & $\begin{array}{l}\text { (1) Le deversoir } \\
\text { commence a fonc } \\
\text { tionner. } \\
\text { (2) Le barrage se } \\
\text { rompt. }\end{array}$ \\
\hline
\end{tabular}

Les volumes d'eau contenus dans le réservoir à l'amont du barrage en fonction de la hauteur du plan d'eau au-dessus du thalweg au droit du barrage étant connus, on a pu établir des graphiques, qui doment, le temps étant porté en abscisse :

Courbe I. - Les hauteurs du plan d'eau;

Courbe II. - Les volumes d'eau contenus dans le réservoir;

Courbe 111. - Le débit total du déversoir et des évacuateurs;

Courbe IV. - Le débit instantané des quantités d'eau, non évacuées avant la rupture du barrage.

Courbe V. - Le débit total de la crue en mètres cubes seconde.

Ces graphique ne sont pas rigoureusement exacts à tout instant parce que les observations des hauteurs du plan d'eau ont ćté faits, au début tout au moins, à des intervalles de temps trop éloignés.

Ils sont cependant assez précis pour la journéc du 26 novembre entre 6 heures du matin et le moment où le barrage s'est rompu.

\begin{tabular}{|c|c|c|c|}
\hline Désignation & $\begin{array}{c}\text { Projet } \\
\text { de } \\
1867\end{array}$ & $\begin{array}{c}\text { Projet } \\
\text { de } \\
1883\end{array}$ & $\begin{array}{l}\text { MonTÉ ANORMALE } \\
\text { du plan d'eau do à un } \\
\text { engorgement du déversoi } \\
\text { provoqué par un espace- } \\
\text { ment trop faible des } \\
\text { fermettes de la } \\
\text { passerelle }(1900) \text {. }\end{array}$ \\
\hline Crête du déversoir....... & $32 \mathrm{~m}$ & $32 \mathrm{~m}$. & $32 \mathrm{~m}$. \\
\hline Lame d'eau déversante... & $1 \mathrm{~m} .60$ & $2 \mathrm{~m} .25$ & $3 \mathrm{~m} .22$ \\
\hline $\begin{array}{r}\text { Cote maximum du plan } \\
\text { deau ............... }\end{array}$ & $33 \mathrm{~m} .60$ & $34 \mathrm{~m} .25$ & $35 \mathrm{~m} .22$ \\
\hline $\begin{array}{l}\text { Débit correspondant des } \\
\text { ouvrages d'évacuation. }\end{array}$ & $\begin{array}{l}570 \mathrm{~m}^{3} \\
\text { seconde }\end{array}$ & $\begin{array}{l}900 \mathrm{~m}^{3} \\
\text { seconde }\end{array}$ & $\begin{array}{l}1.450 \mathrm{~m}^{3} \\
\text { seconde }\end{array}$ \\
\hline
\end{tabular}

De ces graphiques on déduit :

$1^{\circ}$ Que la très forte crue a commencé pratiquement à $20 \mathrm{~h} .30$; $2^{\circ}$ Que le réservoir a été rempli au-dessus de la cote $(20,30)$ jusqu'à la cote $(35,85)$ en 15 heures; 
$3^{\circ}$ Qu'entre le 25 novembre 1927 , à 20 h. 30 et le 26 novembre 1927, à 11 h. 20 (rupture du barrage) il est passé en 15 heures au aroit du barrage 55 millions de metres cubes d'eau dont 20 ont ćté emmagasinés dans le réservoir avant la rupture de l'ouvrage;

$4^{0}$ Que dans celte même période de 15 heures le débit moyen a été de 1.000 mètres cubes seconde, le débit maximum vraiscmblablement atteint au moment de lat rupture du harrage étant de $2.300 \mathrm{~m}^{3}$ seconde.

Ici encore on constate une crue exceptionnelle, notablement plus forte que colles qui correspondent aux plans d'eau maxima prévus en 1867 et en 1883 ou observées en 1900. Les caractéris. tiques de ces crues sont données par le tableau de la page 104.

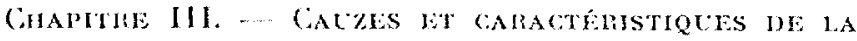 RCPTCRE.}

\section{I. -..- Elat du barrage amant la raphare dil 26 novembre 192.}

$\mathrm{Si}$ on suit le couromement du harrage dans sa longueur de 33.4 metres, depuis la rive droite jusqu'd la rive gauche, on rencontre successivement une série de tronçons qu'on peut distinguer soit par leur orientation en plan, soil par leur profil transversal.

$7^{\text {er }}$ Ironcon. - Sur 30 mìtres environ règne un mur en retour dit " petit mur " clirigé vers l'amont pour atteindre une zone résistante du terrain d'appui. l'arète amont du couromement de ce mur faisait en plan, un angle de $120^{\circ}$, avec l'arête amont du couronnement du "grand mur". Ce petit mur, atteint par l'accident de 1881 , avait éte détruit sur une hauteur qui décroissait de $8 \mathrm{~m}$. 60 ạu sommet de l'angle à 0 à 9 mètres environ de ce sommet: il avait été rétabli, mais épaulé à l'aval en prolongeant le grand mur jusqu'à la rive droite où il était profondément ancre.

20 tronşon. - Sur 129 mètres aul delà de ce sommet, le grand nur du harrage ctait formé d'un soubassement exécuté avant 1870 , surmonté d'un massif reconstruit de 1883 à 1885 , sauf sur quelques mètres à partir du sommet où l'ouvrage avait été reconstruit intégralement y compris la fondation emportée en 1881.

La section longitudinale de la breche de 1881 montre que la profondeur maximum de cette brèche se trouvait au voisinage immédiat de la rive droite, qu'elle déeroissait très légèrement sur près de 90 mètres, à mesure qu'on se rapprochait de la rive gauche. pour diminuer ensuite tres rapidement et se réduire à 0 à 129 metres du sommet de l'angle du grand et du petit mur.

Louvrage avait été rétabli à sa hauteur primitive; les maçonneries du soubassement conservé avaient été taillées en redans, de manière à assurer l'accrochage de la superstructure nouvelle sur la base anciome. Pour renforcer la reprise entre des massifs superposés d’àge different. on avait exécuté au mortier de ciment toutes les maçonneries de liaison jusqu'à un niveau correspondant sensiblement, pour chaque profil, au point culminant de la brèche : il existait donc, dans chaque profil, au-dessus de la surface de reprise entre la maconneric ancienne à la chaux et la maçonnerie nouvelle au ciment, une surface sensiblement horizontale suivant laquelle se faisait le raccord entre la maçonnerie nouvelle au ciment et la maçonnerie nouvelle à la chaux hydraulique qui montait jusqu' au couronmement.

On avait profité de la reconstruction pour améliorer la stabilité de poids du barrage, non séulement en augmentant légèrement sa section transversale, mais encore en redressant le profil vers l'amont, de manière à déplacer le ce côté, le point d'application de la résultante des forces sur une section horizontale, par suite à réduire le taux de travail à la compression du parement aval et a augmenter celui du parement amont. Ce redressement avait été effectué en atténuant le fruit amont, à partir de la cote 8 sur la plus grande longueur de la superstructure nouvelle et à partir d'une cote supéricure croissante, à mesure qu'on se ramprochait de la rive gauche et que la hauteur le la brèche diminuait; il avait éte limité au pilastre qui créait un decrochement en plan au droit de l'évacuateur rive gauche. An delà, le barrige avait ćté reconstruit avec son profil primitif.

$3^{\mathrm{e}}$ tronçon. - A la suite de la zone séparée, sur 175 mètres et jusqu'au déversoir, subsistait louvage ancien; l'accident de 1881 ayant été attribué principalement à la fondation défectueuse du barrage vers la rive droite. on n'avait pas jugé nécessaire de renforcer systématiquement le profil de l'ouvrage dans toute la partic qui u'avait pas été profondément réduite par l'ouverture de la brèche.
En plan, ce $3^{\mathrm{e}}$ tronçon se compose de deux alignements droits formant un angle de $176^{\circ} 30^{\prime}$ saillant vers l'amont, à 125 mètres de la naissance du déversoir.

$4^{\mathrm{e}}$ Ironçon. - Le $4^{\mathrm{e}}$ tronçon comprend le déversoir qui fait en plan avec le barrage un angle de $145^{\circ}$ environ, saillant vers l'aval ; le déversoir est tel qu'il a été rétabli en 1872.

Depuis Ia réparation de $1883-1885$, le barrage n'avait manifesté aucune trace de fatigue anormale. Le parement aval était recouvert de dépôts calcaires provenant de l'entraînement de la chaux par les eaux d'infiltration. Dans le $2^{\mathrm{e}}$ tronçon du barrage, la limite supérieure de cette couche blanche paraissait correspondre sensiblement au niveau supérieur des anciennes maçonneries conservées ; à partir du pilastre, elle montait beaucoup plus haut et suivait une ligne horizontale vers la cote 25 , I) la notice de Pochet sur la construction du barrage, publiée par les Annales des Ponts et Chaussées de 1875 , il résulte que dès la mise en eau du réservoir, des suintements abondants s'étaient produits provoquant la formation d'une couche calcaire sur le parement aval.

L'affaiblissement des mortiers dû à une action prolongée des caux d'infiltration, n'avait pas été sans inquiéter l'Administration qui avait approuvé un projet comportant : d'abord, l'exécution d'injections de ciment à partir du parement amont pour apprécier l'importance des vides existant dans l'ouvrage et pour les boucher éventuellement, ensuite, la pose d'un enduit sur ce parement pour empècher les infiltrations de continuer. Les travaux d'injections en cours sur la rive gauche au moment où l'accident s'est produit et comme la quantité de coulis absorbée était partout relativement faible, on pouvait en déduire que le massif n'était intérieurement ni fracturé, ni caverneux.

On n'avait jamais signalé de fissure sur le parement amont; celui-ci était d'ailleurs depuis longtemps caché sur une partie de sa hauteur par les apports solides qui s'étaient déposés au pied du barrage. Ces dépóts sont constitués par une vase sablonneuse très compacte qui tient naturellement avec un talus presque vertical lorsqu'on l'entaille dans les parties découvertes par l'eau. Cette vase, par conséquent, exerçait sur le pied du barrage une poussée inférieure à la poussée de l'eau.

En reportant sur l'élévation du barrage, les niveaux approximatifs des dépôts à l'amont et du terrain à l'aval, on se rend compte que la hauteur de la tranche supérieure du barrage soumise aux efforts dus à la poussée liquide diminuait rapidement à mesure qu'on s'éloignait de l'évacuateur rive gauche, tandis qu'augmentait celle de la tranche inférieure dont la stabilité de poids était améliorée, d'une part, à cause de la diminution de poussée due à la vase sablonneuse et d'autre part, à cause de l'augmentation de charge verticale due au poids de cette vase sur le talus amont du barrage.

Le soulagement dû à l'amoncellement de vase était d'autant plus sensible que celle-ci était plus sèche. Or, pendant tout l'été 1927, qui a été excessivement chaud, le plan d'eau du réservoir n'a pas dépassé la cote 20 et depuis le milieu d'octobre, il a été maintenu aux environs de la cote 8 . Comme la fin du mois d'octobre et le début du mois de novembre ont été marqués par des températures élevées, non seulement, le fond de la cuvette aux abords du barrage était desséché, sauf dans la zone des évacuateurs, mais encore les maçonneries de la tranche supérieure du barrage exposées pendant plusieurs mois au soleil sans être immergées avaient sans doute perdu la plus grande partie de leur eau d'imbibition. Il est difficile de se faire une idée précise de l'allégement qui a pu résulter de cette dessiccation; si on tient compte de ce que les mortiers étaient revenus plus poreux à la suite des infiltrations qui les avaient altérés et que les pierres de grès employées à la maçonnerie étaient considérées comme notablement poreuses, ainsi que l'indique Pochet dans sa notice, on peut en conclure que, surtout dans la zone amont de l'ouvrage exposée au sud, la diminution du poids unitaire des maçonneries et par conséquent de la stabilité de l'ouvrage a pu être sensible.

\section{II. - Circonstances ayant précédé la rupture.}

Les courbes de la crue pendant les journées des 25 et 26 novembre accusent sa violence exceptionnelle qui se traduit pour le barrage par une ascension extrèmement rapide et anormale du plan d'eau, qui est monté en 11 heures de la cote 8 à la cote 32. crète du déversoir; dès que celui-ci a fonctionné, la montée a été plus lente, mais on peut remarquer qu'entre le moment où le remplissage du réservoir a vraiment commencé $(25$ novembre, 13 heures), et le moment où le barrage s'est rompu (29 
novembre, 11 h. 20), le plan d'eau s'est élevé en 22 h. 20 , de $27 \mathrm{~m} .85$.

Du fait de cette montée extrèmement rapide, les maconneries baignées par l'eau n'ont pas eu le temps de s'imbiber a nouveau. En outre, dans toute la matinée du 26 novembre et. surtout avant la rupture, le débit évacué par le déversoir a été si élevé qu'il a dù régner près du barrage un courant qui a contrarié la décantation de la vase, de telle sorte que pendant cette période la tranche supérieure du barrage située au-dessus des dépôts était soumise, non pas à la poussée de l'eau, mais a celle, plus forte, d'un liquide plus lourd. On n'a pas pu déterminer sa teneur en vase, mais d'apris les renseignements qu'on possède sur le débit solide de l'oued el Hammam et d'aprés les résultats de prélèvements qui ont été opérés les 27 et 28 novembre, il n'est pas exagéré d'admettre qu'aux abords du barrage, l'eau contenait au moins $30 \%$ de vase ce qui donne pour le liquide exerçant la poussée, une densité voi: ne de 1,3 .

Ainsi, à cause de la violence exceptionnelle de la crue survenant brusquement après une période sèche, la stabilité du barrage au moment où s'est produite la rupture était amoindrie parce qu'en mème temps. que la charge verticale résultant du poids de maçomneries supérieures était réduite, particulièrement vers l'amont, la poussée horizontale était notablement augmentée. du fait de la quantité de vase en suspension près du barrage.

\section{III. - Conditions suivant lesquelles s'est produite lä rupture.}

Il est rappelé que la rupture s'est produite le 26 novembre feste près de l'évacuateur rive droite et un sccond plus faible dix mètres au delà; ensuite le talus de la brèche est plus rapide jusqu'à la rencontre du petit mur en retour où la cassure est à peu près verticale.

Vers la rive gauche la profondeur de la brèche diminue d'abord tres peu sur 60 mètres environ, approximativement jusqu'au point oi larete amont de la breche rencontre lin couche de vase déposée à l'amont, et où apparait un décrochement assez brusque de plus de 5 metres; ensuite la pente est faible juscue vers le sommet de l'angle de $176^{\circ}$ formé en plan par le barrage, où existe un décrochement d'environ decix metres; au dela la coupure amont, sensiblement horizontale sur 38 metres environ, se prolonge dans la partie conservée de l'ouvage par une fissure qui regne sur 35 metres dans le parement amont.

En dressant plusieurs profils en travers de la brèche existante et en les rapprochant de ceux qui sont relatifs a la brèche de 1881 , on est tout d'abord frappé de ce que, dans la zone de l'evacuateur de gauche où s'est anorcé la breche, la ligne de rupture le 1927 est un peu en dessous, mais très voisine, de celle de 1881; sette coincidence dome a penser que le barrage a d'abord céde vers la reprise des anciennes at des nouvelles maconneries. peut-itre au voisinage du pilastre dans la partie où on avait maintenu l'amcien profil.

On constate en outre que la brèche au droit du dernier échelon qui s'étend sur la rive gauche et la fissure qui lui fait suile solit ouvertes à un niveau qui correrpond à peu près au sommet de l'angle formé sur le parement aval à l'origine du fruit.

Entin on remarque que d'une maniere générale la brisure
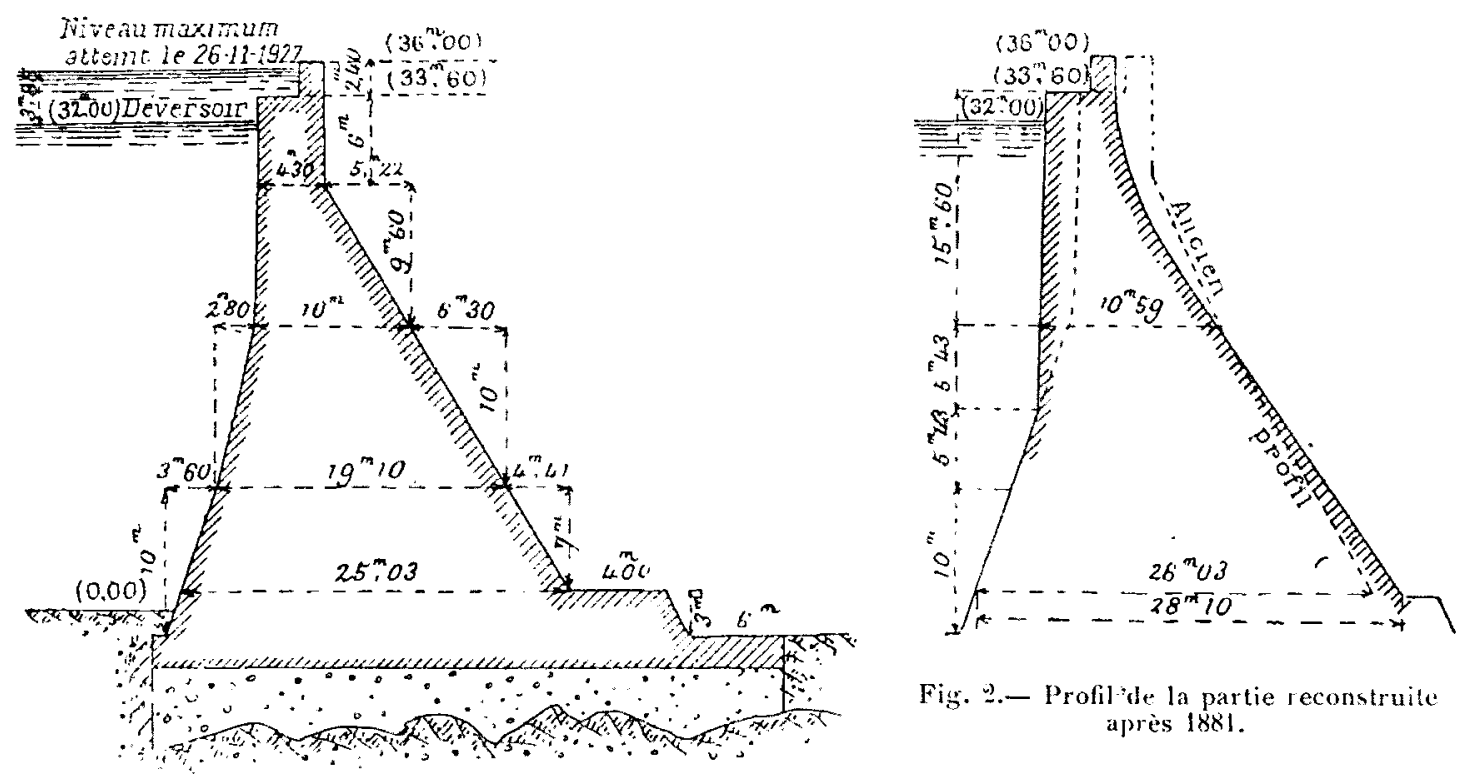

Fig. D.- Profilde la partie reconstruite après 1881 .

Fig. 1.- Profil-type avant la rupture de 1881.

Fig. 1 et 2.- Coupes transwersales du barrage de l'Oued Fergoug.

1927, à 11 h. 20 minutes, alors que le plan d'eau atteignait dans le réservoir au droit du barrage environ la cote 35,85 .

MM. Arargues, Ingénieur des Travaux publies de l'Etat; Bucher, Barragiste; Grisosi, Surveillant des travaux d'injections et de dévasement, témoins oculaires de la rupture, se trouvaient tous les trois sur la rive gauche, la passerelle surmontant le déversoir ayant été emportée, il leur était d'ailleurs impossible d'accéder sur le barrage et ils n'ont pu observer sa ruine que de loin.

Etant donné la soudaineté de la rupture et la position qu'ils occupaient au moment où elle s'est produite, la seule constatation, unanime d'ailleurs, qu'on puisse retenir de leurs déclarations verbales et de leurs dépositions écrites est que la brèche s'est amorcée clans la zone voisine de l'évacuateur de gauche et qu'elle s'est ensuite étendue de part et d'autre dans un temps très court.

\section{IV. - Etal du barrage après la rupture.}

Le plan coté de la brèche a été établi. La section longitudinale de cette brèche au droit du parement amont reportée sur l'élévation amont de l'ouvrage montre que la profondeur de la brèche est maximum au voisinage de l'évacuateur rive gauche. Vers la rive droite un premier décrochement sensible se mani- suivant une section transversalc présente la mème forme qu'en 1881; elle est sensiblement horizontale ou légèrement ascendante jusqu'à une certaine distance du parement amont et clle plonge ensuite vers l'aval avec une inclinaison croissante qui va jusqu'à atteindre par endroit celle du parement aval.

L'examen détaillé des maçomneries encadrant la brèche aprìs les arrachements qui ont accompagné la rupture et le passage prolongé de la lame qui a surmonté l'ouvrage ne peut donner une idée précise de leur état avant laccident; toutefois on n'apercoit pas, entre les pierres, de vides nombreux et importants qui soient de nature à laisser supposer une exécution défectueuse.

Mais dans les coupes apparentes des couches de mortier et surtout au voisinage du parement amont près de l'évacuateur rive gauche, on distinguc de multiples rides aui sont les traces de canaux par lesquels l'cau d'infiltration avait cheminć à travers l'ouvrage; des échantillons de mortier prélevés dans ees couches très délavées se réduisent en poudre si on les presse fortement entre les doigts; d'autres échantillons pris plus à l'aval sont moins appauvris et par suite plus résistants, mais paraissent aussi avoir perdu une bonne partic de leur liant. Les chlorures et les sultates que contient l'eau de l'oued el Hammam ont pu contribuer daus une certaine mesure à cette cuvre d'altération des mortiers.

Il résulte en toul cas de ces constatations que par places et sur- 
tout près du parement amont du barrage, dans la zone où s'est amorcée la rupture, les mortiers étaient notablement affaiblis.

\section{V. - Causes de la rupture.}

Si on considère même le profil renforcé au droit de la partic la plus profonde de la brèche, et si on fait le calcul des efforts qui se produisaient aux extrémites d'une section horizontale correspondant au niveau inférieur de la brèche (cote 12,30) lorsque le plan d'eau dans le réservoir a atteint la cote 35,85 , en supposant que la densité des maçomneries est de 2090, sans tenir compte de la réduction résultant de leur dessication et la densité du licpuide exerçant la poussée est seulement de 1,2, c'est-à-dire en se plaçant dans des conditions plus favorables à la stabilité que celles qui se sont effectivement réalisées au moment de la rupture, on trouve que sur le parement amont, il y avait un effort de traction supérieur à 1 kilo par $\mathrm{cm}^{2}$.

Le mortier étant incapable de résister à cette traction, il a dù se créer dans cette zone particulièrement affaiblie une fissure longitudinale; dès que celle-ci s'est ouverte, l'eau y a pénétré et exercé une sous-pression qui a eu pour effet de diminuer encore la stabilité de la partie supérieure du barrage ; la fracture d'amont, une fois commencée, n'a pu que s'accentuer sous l'action de l'effort de soulèvement qui croissait à mesure que la fissure s'étendait davantage à l'intéricur de l'ouvrage; le travail augmentant en même temps à laval a bientôt dépassé la limite de résistance des maçonneries et la tranche supérieure détachée a été renversée vers l'aval et emportéc par le flot. La dislocation amorcée dans la zone de l'évacuateur de gauche s'est immédiatement développée de part et d'autre avec des décrochements qui correspondent seusiblement à des solutions de continuiti en plan ou en profil c'est-ì-dire à des changements dans les conditions de résistance et de stabilité de l'ouvrage.

D'aprés les résultats de l'étude des documents que la Commission a pu réunir, il ressort bien que le barrage de l'oued Fergoug a été victime d'un concours imprévisible de circonstances défavorables. Sans doute les calculs de stabilité établis pour la charge maximum d'eau qu'on pouvait considérer jusqu'à ces derniers temps comme admissible montrent que le profil de l'ouvage, déterminé d'après les conceptions anciennes, n'offrait pas, mème dans la partie renforcée, les garanties que comportent les théories nouvelles sur les barrages poids et que procurent désormais les formes qu'on leur donne, les moyens qu'on emploie pour les construire et les précautions qu'on prend lors de leur établissement pour les conserver, notamment pour les protéger contre les infiltrations.

Si le barrage de l'oued Fergoug, comme d'ailleurs quelques autres vieux barrages encore en service, ne présentait pas un coefficient de sécurité élevé, il faut reconnaitre cependant qu'il ne s'est brisé qu'après avoir été soumis brutalement à des efforts dépassant de beaucoup ceux auxquels on pouvait s'attendre.

\section{[ EN RÉsUMÉ.}

Le barrage de l'oued Fergoug, brisé une première fois en 1881, avait été rétabli peu de temps après suivant les errements de l'époque avec un profil transversal qui ne réservait pas une notable marge de sécurité ; exposé depuis 45 ans à des infiltrations qui appauvrissaient les mortiers, la résistance de ses maçonneries était amoindrie surtout dans la zone amont. Aussi n'a-t-il pas pu supporter sans se rompre la surcharge que lui a imposée une crue formidable trois fois plus forte que celle pour laquelle il avait été primitivement calculé, survenue après une longue période sèche et qui a provoqué une surélévation extraordinaire du niveau de la retenue avec une eau fortement chargée de vase.

\section{Le Président de la Commission,}

MONET,

Inspecteur général des Ponts et Chaussées.

Président de la $2^{\mathrm{e}}$ section du Conseil Général des Ponts et Chaussées et de la

Commission des Grands Barrages.

Les Rapporteurs,
BOLLARD,
Drouhin,
Ingénieur des Ponts
Ingénieur des Ponts

ct Chaussées.

(Extrait des Annales des Ponts el Chaussées).

\section{Une nouvelle centrale hydro-électrique américaine (électricité et mécanique)}

Cette supercentrale, située ì Conowingo, sur la rivière Susquehanna, sera la plus importante d'Amérique aprés celle des chutes du Niagara. En effet, cette dernière, malgré ses 452.500 chevaux sera largement dépassée par la nouvelle usine dont la puissance, d'abord de 350.000 chevaux atteindra par la suite 550.000 chevaux.

L'usine de Conowingo est construite à $6 \mathrm{~km}$. 500 de la prise d'eau en riviere. Un barrage de 1.464 mètres de longueur placé en travers de la vallée, réalise un immense réservoir d'eau de 3.280 hectares.

L'eau captée actionnera, dès le début, sept génératrices de $36.000 \mathrm{KW}$ au moyen de sept turbines hydrauliques géantes de 50.000 chevaux et l'énergie électrique produite sera transportée à très haute tension à Philadelphie, soit à 120 kilomètres de là. les alternateurs construits actuellement dans les ateliers de la Général libectric Company, sont privus pour une puissance de $10.000 \mathrm{KV}-\mathrm{A}$, avec un facteur de puissance de 0,9 , une tension de 13.000 volts et une vitesse de $81,8 \mathrm{t} / \mathrm{m}$., sous me chute de 27 mètres. La centrale de Conowingo pourra produire, d'après les estimations déjà faites, environ 1 milliard 360 millions de KW-H. par an, d'où une économie anmuelle de houille de 750.000 tonnes par an. Elle viendra en aide de la Centrale de Holtwood, élevée à $24 \mathrm{~km}$. en aval, qui, sous une chute de $18 \mathrm{~m}$. fournit déjà $500 \mathrm{mil}$ lions de KW-H. par an à la ville de Baltimore, située à $88 \mathrm{~km}$. plus au sud.

Le lit de la rivière, etant en granit, assure un barrage de très solides fondations et permet d'envisager à $32 \mathrm{~m}$. all-dessus de ces fondations la construction du pont de la route principale de Baltimore à l'hiladelphie. Ce pont remplacera le pont actuel qui est englobé dans laaire du réservoir. Le réservoir sera situé dans la partic Est ih barrage, tandis que le bâtiment de la centrale sera à l'ouest.

La crête du réservoir atteindra la cote de $26 \mathrm{~m}$. au-dessus du niveau de la mer : ce réservoir comportera 50 vannes mobiles en acier cle $6 \mathrm{~m} .68$ de hauteur et $12 \mathrm{~m} .20$ de largeur ; elles maintiendront le nivcau à la cote de $33 \mathrm{~m}$. Quand toutes les vannes seront ouvertes, le réservoir pourra évacuer un volume d'eau de $23 \mathrm{~m}^{3} / \mathrm{sec}$.

Le bâtiment de la centrale aura $53 \mathrm{~m}, 40$ de large sur $190 \mathrm{~m}$. de long. Cette dernière dimension pourra être portée ultérieurement à 275 mìtres.

Du fond des conduites forcées jusqu'au faite de la station de coupure à haute tension qui sera établie sur le toit de la centrale, on prévoit une hauteur de 70 mètres. Notons, en passant, que le fond des conduites forcées sera à $7 \mathrm{~m}$. 60 en dessous du niveau de la mer.

Les génératrices produiront le courant à la tension de $13.800 \mathrm{v}$. que des tramsformateurs élèveront à 220.000 volts. Deux lignes alimenteront Philadelphie, mais chacune sera capable de transporter la totalité du courant. Plus tard, quand les groupes générateurs $N^{\text {os }} \$, 9,1 \$$ et 11 auront été installés, une troisième ligne de transport sera mise en chantier.

Le bassin de la rivière Susquehanna, dont la superficie est de $70.000 \mathrm{~km}$. carrés, comprend une grande partie de l'Etat de Pensylvanie, une portion considérable de l'Etat méridional de NewYork et une fraction du nord-est de l'Etat de Maryland.

Le débit moyen de la rivière est de 1.132 litres à la seconde. L'installation hydro-electrique de Conowingo pourra fonctionner conjointement avec des centrales thermiques. En période de gros débit, Conowingo fournira la charge principale du réseau, les centrales thermiques entrant en jeu au moment des pointes, et inversement. Avec les sept premières unités, la centrale de Conowingo pourra produire une puissance hydraulique de $231.000 \mathrm{kw}$, qui formeront la partic principale de la charge. En période d'étiage, on fera appel au vaste réservoir mentionné plus haut; des statis- 